\title{
Blow up Dynamic and Upper Bound on the Blow up Rate for critical nonlinear Schrödinger Equation
}

\author{
Frank Merle
}

\author{
Pierre Raphael
}

Abstract

We consider the critical nonlinear Schrödinger equation $i u_{t}=-\Delta u-$ $|u|^{\frac{4}{N}} u$ with initial condition $u(0, x)=u_{0}$ in dimension $N$. For $u_{0} \in H^{1}$, local existence in time of solutions on an interval $[0, T)$ is known, and there exists finite time blow up solutions, that is $u_{0}$ such that $\lim _{t \rightarrow T<+\infty}\left|u_{x}(t)\right|_{L^{2}}=+\infty$. This is the smallest power in the nonlinearity for which blow up occurs, and is critical in this sense. The question we address is to understand the blow up dynamic. Even though there exists an explicit example of blow up solution and a class of initial data known to lead to blow up, no general understanding of the blow up dynamic is known. At first, we propose in this paper a general setting to study and understand small in a certain sense blow up solutions. Blow up in finite time follows for the whole class of initial data in $H^{1}$ with strictly negative energy, and one is able to prove a control from above of the blow up rate below the one of the known explicit explosive solution, which has strictly positive energy.

We consider the critical nonlinear Schrödinger equation

$$
\begin{cases}i u_{t}=-\Delta u-|u|^{\frac{4}{N}} u, & (t, x) \in[0, T) \times \mathbb{R}^{N} \\ u(0, x)=u_{0}(x), & u_{0}: \mathbb{R}^{N} \rightarrow \mathbb{C}\end{cases}
$$

with $u_{0} \in H^{1}=H^{1}\left(\mathbb{R}^{N}\right)$, in dimension $N \geq 1$. This equation is locally well-posed in $H^{1}$ from [2]. The problem we address is the one of formation of singularities for solutions to (NLS). Note that from the conservation of the mass and the energy (from the Hamiltonian formulation) and Gagliardo-Nirenberg inequality, the power of the nonlinearity is the smallest one for which blow-up may occur. We will see that this criticality makes the problem global.

In the energy space $H^{1}$, (NLS) admits three conservation laws: $L^{2}$-norm, Energy, Momentum, and four fundamental symetries: Space-time translation, Phase, Scaling and Galilean invariances.

At the critical power, special regular solutions play an important role. They are the so called solitary waves and are of the form $u(t, x)=e^{i \omega t} W_{\omega}(x), \omega>0$, where $W_{\omega}$ solves

$$
\Delta W_{\omega}+W_{\omega}\left|W_{\omega}\right|^{\frac{4}{N}}=\omega W_{\omega} .
$$


Equation (1) is a standard nonlinear elliptic equation. There is a unique positive solution up to translation $Q_{\omega}(x) . Q_{\omega}$ is in addition radially symmetric. Letting $Q=Q_{\omega=1}$, scaling properties and Pohozaev identity yield $\left|Q_{\omega}\right|_{L^{2}}=|Q|_{L^{2}}$ and $E\left(Q_{\omega}\right)=0$. In particular, none of the three conservation laws in $H^{1}$ sees the variation of size of the $W_{\omega}$ stationary solutions. These two facts are deeply related to the criticality of the problem. Weinstein in [9] used the variational characterization of the ground state solution $Q$ to (1) to derive the explicit constant in the GagliardoNirenberg inequality

$$
\forall u \in H^{1}, \quad \frac{1}{2+\frac{4}{N}} \int|u|^{\frac{4}{N}+2} \leq \frac{1}{2}\left(\int|\nabla u|^{2}\right)\left(\frac{\int|u|^{2}}{\int Q^{2}}\right)^{\frac{2}{N}},
$$

so that for $\left|u_{0}\right|_{L^{2}}<|Q|_{L^{2}}$, the solution is global in $H^{1}$. In addition, blow up in $H^{1}$ has been proved to be equivalent to "blow up" for the $L^{2}$ theory from concentration in $L^{2}$.

On the other hand, for $\left|u_{0}\right|_{L^{2}} \geq|Q|_{L^{2}}$, blow up may occur. Indeed, for this special power nonlinearity, (NLS) admits another symmetry which is not in the energy space $H^{1}$, the so called pseudo conformal transformation: if $u(t, x)$ solves (NLS), then so does $v(t, x)=\frac{e^{i|x|^{2} / 4 t}}{|t|^{N / 2}} \bar{u}\left(\frac{1}{t}, \frac{x}{t}\right)$. This additional symmetry yields for $u_{0} \in \Sigma=H^{1} \cap\left\{x u \in L^{2}\right\}:$

$$
\frac{d^{2}}{d t^{2}} \int|x|^{2}|u(t, x)|^{2}=4 \frac{d}{d t} \operatorname{Im}\left(\int x \nabla u \bar{u}\right)(t, x)=16 E\left(u_{0}\right) .
$$

Now since $E(Q)=0$ and $\nabla E(Q)=-Q$, there exists $u_{0 \epsilon} \in \Sigma$ with $\left|u_{0 \epsilon}\right|_{L^{2}}=|Q|_{L^{2}}+\epsilon$ and $E\left(u_{0 \epsilon}\right)<0$, and the corresponding solution must blow up from viriel identity (3).

The case of critical mass $\left|u_{0}\right|_{L^{2}}=|Q|_{L^{2}}$ has been studied by Merle in [5]. The pseudo-conformal transformation applied to the stationary solution $e^{i t} Q(x)$ yields an explicit solution

$$
S(t, x)=\frac{e^{i \frac{|x|^{2}}{4 t}-\frac{i}{t}}}{|t|^{\frac{N}{2}}} Q\left(\frac{x}{t}\right)
$$

which blows up at $T=0$. Note that $|S(t)|_{L^{2}}=|Q|_{L^{2}}$. It turns out that $S(t)$ is the unique minimal mass blow up solution in $H^{1}$ in the following sense: let $u(-1) \in H^{1}$ with $|u(-1)|_{L^{2}}=|Q|_{L^{2}}$, and assume that $u(t)$ blows up at $T=0$, then $u(t)=S(t)$ up to the symmetries of the equation.

Another fact suggested by numerical simulations, see [3], is the existence of solutions blowing up as

$$
|\nabla u(t)|_{L^{2}} \sim \sqrt{\frac{\ln |\ln | t||}{|t|}} .
$$

These appear to be stable with respect to perturbation of the initial data. In this frame, for $N=1$, Perelman in [8] proves the existence of one solution which blows up according to (5). Note that such solutions are stable with respect to perturbation of the initial data from numerics, but are known to be structurally unstable. Indeed, in dimension $N=2$, if we consider the next term in the physical approximation 
leading to (NLS), we get Zakharov equation, and finite time blow up solutions to Zakharov equation are known to satisfy $|\nabla u(t)|_{L^{2}} \geq \frac{C}{|T-t|}$.

Our approach to study blow up solutions to (NLS) is based on a qualitative description of the solution as in [4]. We focus on the case when the nonlinear dynamic plays a role and interacts with the dispersive part of the solution. This last part will be proved to be small in $L^{2}$ for initial conditions which satisfy

$$
\int Q^{2}<\int\left|u_{0}\right|^{2}<\int Q^{2}+\alpha_{0} \text { and } E\left(u_{0}\right)<0
$$

where $\alpha_{0}$ is small. Indeed, under assumption (6), from the conservation laws and the variational characterization of the ground state $Q$, the solution $u(t, x)$ remains close to $Q$ in $H^{1}$ up to scaling and phase parameters, and also translation in the non radial case. We then are able to define a regular decomposition of the solution of the type

$$
u(t, x)=\frac{e^{i \gamma(t)}}{\lambda(t)^{\frac{N}{2}}}(Q+\epsilon)\left(t, \frac{x-x(t)}{\lambda(t)}\right)
$$

where $|\epsilon(t)|_{H^{1}} \leq \delta\left(\alpha_{0}\right)$ with $\delta\left(\alpha_{0}\right) \rightarrow 0$ as $\alpha_{0} \rightarrow 0, \lambda(t)>0$ is a priori of order $\frac{1}{|\nabla u(t)|_{L^{2}}}, \gamma(t) \in \mathbb{R}, x(t) \in \mathbb{R}^{N}$.

In particular, we derive a control from above of the blow rate for such solutions. More precisely, we claim the following assuming a spectral property on a potential related to $Q$ and checked in dimension $N=1$.

Theorem 1 ([6],[7]) There exists $\alpha^{*}>0$ and $C^{*}$ such that the following is true. Let $u_{0} \in H^{1}$ such that

$$
0<\int\left|u_{0}\right|^{2}-\int Q^{2}<\alpha^{*}, \quad E_{0}<\frac{1}{2}\left(\frac{\left|\operatorname{Im}\left(\int \nabla u_{0} \overline{u_{0}}\right)\right|}{\left|u_{0}\right|_{L^{2}}}\right)^{2} .
$$

Let $u(t)$ be the corresponding solution to (NLS), then:

(i) $u(t)$ blows up in finite time $T<+\infty$ in $H^{1}$.

(ii) Moreover, $|\nabla u(t)|_{L^{2}} \leq C^{*}\left(\frac{\ln |\ln (T-t)|}{T-t}\right)^{\frac{1}{2}}$ holds for $t$ close to $T$.

\section{Comments on the result}

1.Blow up rate: Assume that $u$ blows up in finite time. By scaling properties, a known lower bound on the blow up rate is $|\nabla u(t)|_{L^{2}} \geq \frac{C^{*}}{\sqrt{T-t}}$.

The problem here is to control the blow up rate from above. Our result is the first of this type for critical NLS. No upper bound on the blow up rate was known, not even of exponential type. Note indeed that there is no Lyapounov functional involved in the proof of this result, and that it is purely a dynamical one with all dynamical controls exhibited in $H^{1}$ and not in $\Sigma$.

We first prove an upper bound on the blow up rate as $|\nabla u(t)|_{L^{2}} \leq \frac{C^{*}}{\sqrt{\left|E_{0}\right|}(T-t)}$. This bound is optimal for NLS in the sense that there exist blow up solutions with 
this blow up rate, explicitly $S(t)$ of (4) or more generally those build in [1]. Note nevertheless that these solutions have strictly positive energy.

In our setting of strictly negative energy initial conditions, no solutions of this type is known, and we indeed are able to improve the upper bound by excluding any polynomial growth between the pseudo-conformal blow up and the scaling estimate. It says in particular that there is a large open set of initial data which blow up with a control from above of the blow-up rate suggested by numerics. This bound is conjectured to be optimal.

We would like to point out that this improvement of blow up rate control heavily relies on algebraic cancellations deeply related to the degeneracy of the linear operator around $Q$ which are unstable with respect to "critical" perturbations of the equation. Indeed, recall for example that all strictly negative energy solutions to Zakharov equation satisfy the lower bound $|\nabla u(t)|_{L^{2}} \geq \frac{C}{|T-t|}$. On the other hand, we expect the first argument to be structurally stable in a certain sense.

2. Blow up result: In the situation $\int\left|u_{0}\right|^{2} \leq \int|Q|^{2}+\alpha_{0}$, we show that blow up is related to a local in space information, and we do not need the additional assumption $u_{0} \in \Sigma=H^{1} \cap\left\{x u \in L^{2}\right\}$.

\section{References}

[1] Bourgain, J.; Wang, W., Construction of blowup solutions for the nonlinear Schrödinger equation with critical nonlinearity. Ann. Scuola Norm. Sup. Pisa Cl. Sci. (4) 25 (1997), no. 1-2, 197-215 (1998).

[2] Ginibre, J.; Velo, G., On a class of nonlinear Schrödinger equations. I. The Cauchy problem, general case. J. Funct. Anal. 32 (1979), no. 1, 1-32.

[3] Landman, M. J.; Papanicolaou, G. C.; Sulem, C.; Sulem, P.-L., Rate of blowup for solutions of the nonlinear Schrödinger equation at critical dimension. Phys. Rev. A (3) 38 (1988), no. 8, 3837-3843.

[4] Martel, Y.; Merle, F., Blow up in finite time and dynamics of blow up solutions for the $L^{2}$-critical generalized KdV equation, to appear in J. Amer. Math. Soc.

[5] Merle, F., Determination of blow-up solutions with minimal mass for nonlinear Schrödinger equations with critical power. Duke Math. J. 69 (1993), no. 2, 427-454.

[6] Merle, F.; Raphael, P., Blow up dynamic and upper bound on the blow up rate for critical nonlinear Schrödinger equation, preprint.

[7] Merle, F.; Raphael, P., Sharp upper bound on the blow up rate for critical nonlinear Schrodinger equation, preprint.

[8] Perelman, G., On the blow up phenomenon for the critical nonlinear Schrödinger equation in 1D, to appear in Annale Henri Poincare.

[9] Weinstein, M.I., Nonlinear Schrödinger equations and sharp interpolation estimates, Comm. Math. Phys. 87 (1983), 567-576. 
Université De Cergy-Pontoise

Institut Universitaire DE FranCE 